\title{
Fast Estimation of BER in PAR-Limited DMT Systems Using Noise Injection Method
}

\author{
Behrouz Farhang-Boroujeny, Senior Member, IEEE, Wu Yan, and Samir Attallah
}

\begin{abstract}
High peak-to-average power ratio is a major problem that is inherent in all multicarrier communication systems. Clipping the high-peak power samples introduces (extra) clipping noise, which degrades the system performance in terms of bit-error rate (BER). Measuring BER usually requires a very long simulation time, as clipping occurs very rarely. In this letter, we propose a method called clipping noise injection, which allows estimating BER (due to clipping) in discrete multitone-based systems in a fast and quite precise way. Savings of as great as $\mathbf{9 0 \%}$ or more are observed in some simulations that are reported in this letter. Higher savings are expected when lower BER cases have to be studied.
\end{abstract}

Index Terms-Bit-error rate (BER), clipping noise, discrete multitone (DMT), fast estimation.

\section{INTRODUCTION}

D ISCRETE multitone (DMT) modulation has been standardized as the line code for asymmetric digital subscriber line (ADSL) transceivers. According to the central limit theorem, the time-domain DMT samples have a zero-mean Gaussian distribution [13]. Therefore, it is possible that some samples have an amplitude that is larger than the maximum dynamic range of the digital-to-analog (D/A) and analog-to-digital (A/D) converters or the power amplifier in the system. In this case, clipping occurs at these samples and degrades the system bit-error rate (BER) performance. Since clipping occurs very rarely and becomes even rarer after applying peak-to-average power ratio (PAR)-reduction schemes (see, e.g., [2], [4]-[10]), the BER is very small. Therefore, it is extremely time consuming to measure the BER.

In [1], a fast method is proposed to estimate the error propagation in decision feedback detectors. This method speeds up the simulations by artificially injecting noise samples into the system. The main thrust of this letter is to extend the idea of noise injection to the DMT systems to provide a fast estimate of the BER in some PAR-reduction methods. In this method, noise samples which have the same characteristics and effect as clipping noise are injected at unclipped data symbols in order

Paper approved by Y. Li, the Editor for Wireless Communication Theory of the IEEE Communications Society. Manuscript received August 16, 2001; revised December 15, 2001; March 24, 2002; and April 24, 2002. This paper was presented in part at GlobeCom 2002, Taipei, Taiwan, R.O.C., November 17-21, 2002.

B. Farhang-Boroujeny is with the University of Utah, Electrical Engineering Department, Salt Lake City, UT 84112-9206 USA (e-mail: farhang@ee.utah.edu).

W. Yan is with the Centre for Wireless Communications, Singapore 117674 (e-mail: cwcwuyan@nus.edu.sg).

S. Attallah is with the School of Electrical and Computer Engineering, Curtin University of Technology, Perth 6845, Australia (e-mail: s.attallah@ece.curtin.edu.au).

Digital Object Identifier 10.1109/TCOMM.2003.809225 to observe the effect of clipping more frequently, thus reducing the simulation time significantly.

\section{Probability Distribution of ClipPing Noise}

Assuming the clipping level is $A_{\text {clip }}$, the clipping noise $x_{c}$ for a DMT sample $x$ can be written as

$$
x_{c}= \begin{cases}-\left(x-A_{\text {clip }}\right), & x \geq A_{\text {clip }} \\ -\left(x+A_{\text {clip }}\right), & x<-A_{\text {clip }} .\end{cases}
$$

The probability density function (PDF) of the clipping impulses is

$$
p_{x_{c}}\left(x_{c}\right)= \begin{cases}\left(\frac{1}{P_{t}}\right) \frac{1}{\sqrt{2 \pi} \sigma} \exp \left(-\frac{\left(-x_{c}+A_{\text {clip }}\right)^{2}}{2 \sigma^{2}}\right), & x_{c} \leq 0 \\ \left(\frac{1}{P_{t}}\right) \frac{1}{\sqrt{2 \pi} \sigma} \exp \left(-\frac{\left(-x_{c}-A_{\text {clip }}\right)^{2}}{2 \sigma^{2}}\right), & x_{c}>0\end{cases}
$$

where $P_{t}$ is the cumulative probability of the tail portion of the zero-mean Gaussian distribution, and the factor of $1 / P_{t}$ is introduced so as to normalize the tail portion to a total probability of one. Once we get the distribution function of the clipping impulse from (2), generating the clips becomes straightforward.

Another problem that needs consideration here is how many clips should be injected in each block of DMT samples. To answer this question, we need to calculate the probability of having $n$ clips inside one block of DMT samples. The probability that a DMT sample exceeds the clipping level, $A_{\text {clip }}$, is given by $P_{t}$. To simplify our discussion here and also the noise-injection procedures that are developed subsequently, we only consider the clips which occur at isolated samples of DMT. That is, at samples that are sufficiently apart from each other, so that the clipped samples can be assumed independent of one another. In the next section, where we present more details of the noise-injection method, correlation among neighboring samples is considered and integrated in the subsequent steps of the noise-injection procedures.

In light of the above discussion, the probability of having $m$ isolated clipped samples inside one block of 512 DMT samples can be calculated as

$$
P_{m}=C_{512}^{m} P_{t}^{m}\left(1-P_{t}\right)^{512-m}
$$

where $C_{512}^{m}=512 ! / m !(512-m)$ ! is the combinatorial coefficient. Therefore, the probability of having $n$ isolated clips or more is given by

$$
P(m \geq n)=1-\sum_{i=0}^{n-1} C_{512}^{i} P_{t}^{i}\left(1-P_{t}\right)^{512-i} .
$$

We can also work out the probability of having $n$ isolated clips inside a block of DMT samples given clipping has occurred as

$$
P_{n \mid \text { clip }}=\frac{C_{512}^{n} P_{t}^{n}\left(1-P_{t}\right)^{512-n}}{1-\left(1-P_{t}\right)^{512}} \text {. }
$$


Using (5), the generation of the (isolated) injected clips can be performed as follows:

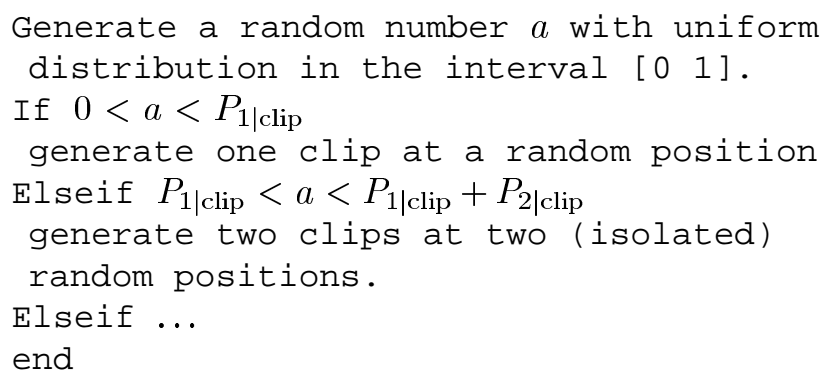

Numerical study of (4) reveals that as $n$ increases, $P(m \geq n)$ decreases very fast and becomes negligible. Thus, it is sufficient to generate a finite number of clips inside every DMT block. For example, if $A_{\text {clip }}=3.5 \sigma$, we find that $P(m \geq 5)=5.152 \times$ $10^{-6}$. Hence, the maximum number of isolated clips we need to generate in each DMT block may be set to four, if we ignore those cases with probability less than $10^{-5}$.

\section{ClipPing Noise InJECTION}

In this section, we introduce the method of clipping noise injection to provide a fast estimation of the BER performance of the DMT-based ADSL systems. In particular, we present a detailed study on clipping noise injection for DMT systems employing a spectrum-shaping method [2] and an optimal-filtering method [4].

\section{A. Overview of Clipping Noise Injection}

As the name suggests, the idea of clipping noise injection is to make clipping present at every DMT block by purposefully injecting clipping noise into each block of DMT samples whenever clipping does not occur. The BER for this scenario will be much larger due to the more frequent occurrence of clipping, hence, a shorter simulation time is needed. The actual BER of the system can then be approximated by multiplying the BER after clipping noise injection with the probability of clipping, $P_{\text {clip }}=\sum_{n} P_{m}$. To include the errors due to channel noise as well, we proceed as follows.

We note that all the DMT blocks fall into two categories, with clipping and without clipping. Let $\mathrm{BER}_{c}$ and $\mathrm{BER}_{n}$ denote the BER due to clipping noise and the BER due to channel noise,

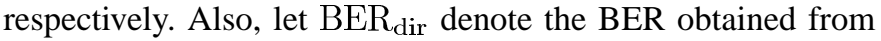
direct simulation. Since under typical operation of DMT, BER and $\mathrm{BER}_{n}$ are small, we assume the error events due to clipping noise and channel noise are exclusive, and accordingly obtain

$$
\mathrm{BER}_{\text {dir }}=\mathrm{BER}_{c} \times P_{\text {clip }}+\mathrm{BER}_{n} \times\left(1-P_{\text {clip }}\right) .
$$

On the other hand, if we assume that $\mathrm{BER}_{c}$ is equal to BER observed through noise injection, i.e., $\mathrm{BER}_{c}=\mathrm{BER}_{\mathrm{inj}}$, from (6), we obtain

$$
\mathrm{BER}_{\text {dir }}=\mathrm{BER}_{\text {inj }} \times P_{\text {clip }}+\mathrm{BER}_{n} \times\left(1-P_{\text {clip }}\right) .
$$

The results presented in Section IV show that (7) provides an accurate estimate of the BER, and thus, confirm the accuracy of the approximations made in its derivation.

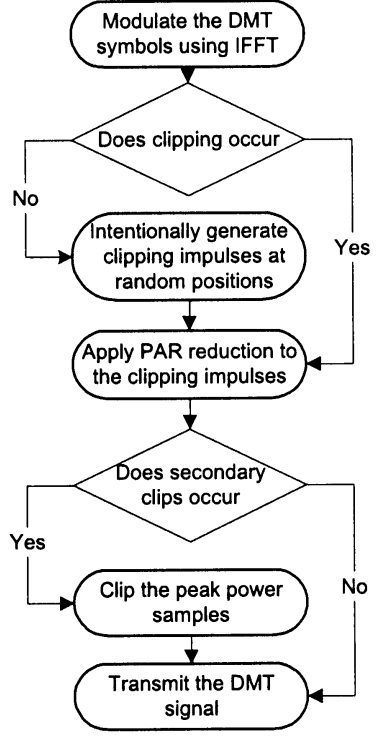

Fig. 1. Procedures of clipping noise injection.

The procedure of noise injection is summarized in Fig. 1. For every DMT symbol, we check whether clipping has occurred or not. If clipping has occurred, we directly apply a PAR-reduction method to the symbol. If clipping has not occurred, we generate clipping impulses with the distribution given in Section II at isolated random positions and add them to the original DMT symbol. We call this step the clipping noise injection. Correlation among DMT samples is then considered and the samples adjacent to the clipped samples are modified accordingly. After that, we apply a clipping-reduction scheme to the injected clips and measure the BER.

\section{B. Noise Injection for Spectrum-Shaping Method}

In [2], Chow et al. proposed the spectrum-shaping method, which employs a simple high-pass filter with impulse response of $\mathbf{h}=[-0.51-0.5]$ to reduce the probability of clipping. In $\mathbf{h}$, the middle sample is used to remove the excessive size of the clipped sample, while the other samples are included to push the clipping noise toward high-frequency subcarriers. For ease of reference, we shall refer to the middle sample as the primary sample and to the rest as secondary samples.

In a DMT-based ADSL system, usually there are no bits allocated to some high-frequency subcarriers because of their poor signal-to-noise ratio (SNR). As a result, the DMT samples after inverse fast Fourier transform (IFFT) are correlated. Therefore, when clipping occurs, it is likely that the neighboring samples of the clipped sample have high amplitudes, too, due to the correlation. As discussed in Section II, we ignore such correlations in the startup of the noise-injection procedure. This, through computer simulations, has been found to be an acceptable approximation. However, when applying spectrum shaping, the probability of getting secondary clips is high and these may not be ignored. Secondary clips refer to the new clips generated while compensating for an existing clip. On the other hand, for a block of DMT samples without any clipping, all the samples are likely to be low in amplitude due to the correlation between them. 
Thus, if we inject clipping noise at random positions and perform filtering directly, the chance of getting secondary clips will be lower compared to direct simulations. Therefore, in order to get the correct number of secondary clips, we must take into consideration the factor of correlation among samples in each DMT block whenever a clip is injected.

Since the high-pass filter used in the spectrum-shaping method of [2] has only three taps, we only need to consider the two adjacent samples of each injected clipping noise sample. Let the vector $\mathbf{x}$ denote a DMT block with none of its samples exceeding the clipping level. We inject a clipping impulse with amplitude $x_{c}$ to a randomly selected sample of $\mathbf{x}, x_{n}$. This simulates the case where the $n$th sample of $\mathbf{x}$ is equal to $A_{\text {clip }}-x_{c}$. Let us denote this new sample as $x_{n}^{\prime}$ to distinguish it from the original $x_{n}$. Due to the correlation between the time-domain samples, the two adjacent samples $x_{n+1}^{\prime}$ and $x_{n-1}^{\prime}$ are likely to have high amplitudes as well, thus, they may be clipped. Given the sample value of $x_{n}^{\prime}$, the conditional distribution of $x_{n+1}^{\prime}$ and $x_{n-1}^{\prime}$ is given by [12]

$$
P\left(x^{\prime} \mid x_{n}^{\prime}=k\right)=\frac{1}{\sigma \sqrt{2 \pi\left(1-r_{1}^{2}\right)}} \exp \left(-\frac{\left(x^{\prime}-r_{1} k\right)^{2}}{2 \sigma^{2}\left(1-r_{1}^{2}\right)}\right)
$$

where $r_{1}$ is the correlation coefficient of $x_{n}^{\prime}$ and $x_{n+1}^{\prime}$ (or $x_{n}^{\prime}$ and $\left.x_{n-1}^{\prime}\right)$. It can be shown that when similar power is allocated to all used subcarriers, the correlation coefficient of $x_{m}$ and $x_{n}$ is given by

$$
r_{m-n}=\frac{\mathbf{f}_{m-n}^{H} \mathbf{p}}{\mathbf{f}_{0}^{H} \mathbf{p}}
$$

where $\mathbf{f}_{i}^{H}$ is the $i$ th row of the inverse discrete Fourier transform (IDFT) matrix, and $\mathbf{p}$ is a vector having values of one in all the used subcarriers and values of zero in the unused subcarriers.

Thus, starting with a random position $n$, we generate the dummy samples $x_{n-1}^{\prime}, x_{n}^{\prime}$, and $x_{n+1}^{\prime}$ as mentioned above. These samples replace $x_{n-1}, x_{n}$, and $x_{n+1}$ in $x$. We call the resulting vector $\mathbf{x}^{\prime}$. We then apply the spectrum-shaping method to $\mathbf{x}^{\prime}$ and clip the secondary clips if they occur. This results in a new vector which is called $\mathbf{x}^{\prime}{ }_{\text {clip }}$. We then define $\mathbf{x}^{\prime}{ }_{c}=\mathrm{x}^{\prime}{ }_{\text {clip }}-\mathrm{x}^{\prime}$. This gives the final clipping noise vector. Therefore, the noise injection of the original DMT vector $\mathrm{x}$ is realized by adding $\mathbf{x}^{\prime}{ }_{c}$ to $\mathbf{x}$. This procedure specifies the injection and filtering of a single clip. The injection and filtering of multiple clips is performed similarly.

\section{Noise Injection for Optimal-Filtering Method}

The procedure for clipping noise injection for the case of the optimal-filtering method proposed in [4] is similar to the case of the spectrum-shaping method introduced above. Here, we are considering an optimal filter with a length of 33 taps, designed using constrained least-square (CLS) method [3]. Therefore, the only difference from the spectrum-shaping case is that we need to consider secondary clips due to 32 neighboring samples of the injected clipping impulse, instead of two.

Thorough study of the correlation coefficient for DMT samples with different lags has revealed that the majority of the secondary clips are likely to occur at the first two neighboring samples of $x_{n}$ [3]. Therefore, we can make the approximation

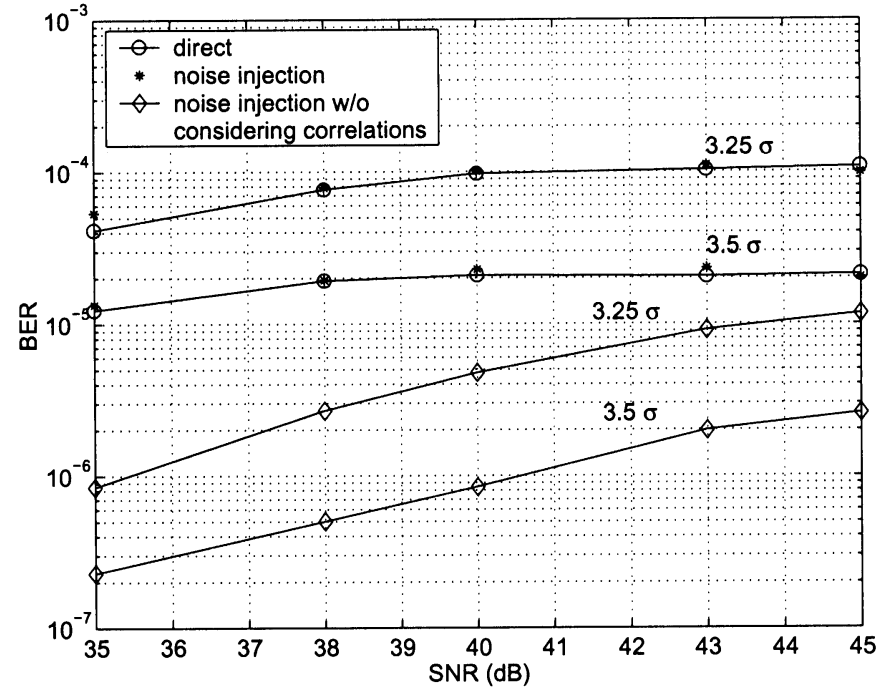

Fig. 2. BER performance of spectrum-shaping method. Results compare direct simulation with clipping noise-injection method. Results of simulations that do not consider correlation among neighboring samples are also presented to show the significance of correlations.

by only considering the first two neighboring samples and assuming that the correlations of the other neighboring samples are zero. Following the same procedure as the clipping noiseinjection method for spectrum shaping, we need to generate dummy samples $x_{n-2}^{\prime}, x_{n-1}^{\prime}, x_{n+1}^{\prime}$, and $x_{n+2}^{\prime}$ given that there is an injected clipping noise at $x_{n}^{\prime}$. The distribution function for $x_{n-1}^{\prime}$ and $x_{n+1}^{\prime}$ given $x_{n}^{\prime}$ is given by (8). Strictly speaking, the distribution of $x_{n-2}^{\prime}$ depends on the values of $x_{n-1}^{\prime}, x_{n}^{\prime}$, and $x_{n+1}^{\prime}$. The same argument applies for $x_{n+2}^{\prime}$ as well. Ignoring the second and higher order dependency of the samples as we did for the spectrum-shaping method, we assume in our simulations that the distribution function of $x_{n-2}^{\prime}\left(\right.$ or $\left.x_{n+2}^{\prime}\right)$ depends only on the value of $x_{n}^{\prime}$, and thus, a similar distribution to (8) is used to select $x_{n-2}^{\prime}$ and $x_{n+2}^{\prime}$.

\section{Simulation Results}

In this section, we present the simulation results for the estimation of the BER of the clipping noise-injection method as well as the BER obtained from direct simulations. We also give the simulation time ${ }^{1}$ required for both methods. The channel model used in all the simulations is loop six of the eight carrier serving area (CSA) loops mentioned in [11]. The bit loading follows Chow's on-off energy-distribution calculation algorithm [13]. For simplicity of simulation, no coding scheme is included in the system. Square constellations are used in all the simulations to simplify mapping and demapping. Channel noise is assumed to be white.

Fig. 2 shows simulation results of BER from the clipping noise-injection method and the BER from the direct simulation for the spectrum-shaping method. Under no clipping noise, for each SNR, bit allocation has aimed for a BER of $10^{-7}$. We observe that the noise-injection procedure produces a good approximation to the actual BER. In Fig. 2, we also show the BER

\footnotetext{
${ }^{1}$ The time is recorded by running simulations on a Pentium III $450-\mathrm{MHz}$ PC
} with 128-MB RAM 
TABLE I

Simulation Time For SPECtRUM-SHAPING MEthod BEFore AND AFTER USING CLIPPING NOISE INJECTION SNR $(=35 \mathrm{~dB})$

\begin{tabular}{c|c|c}
\hline Clipping Level & Direct Simulation & Noise Injection Method \\
\hline $3.25 \sigma$ & $22 \min 54 \mathrm{~s}$ & $8 \min 25 \mathrm{~s}$ \\
\hline $3.5 \sigma$ & $1 \mathrm{~h} 16 \min 16 \mathrm{~s}$ & $16 \min 33 \mathrm{~s}$ \\
\hline $3.75 \sigma$ & $7 \mathrm{~h} 4 \min 5 \mathrm{~s}$ & $35 \min 9 \mathrm{~s}$ \\
\hline
\end{tabular}

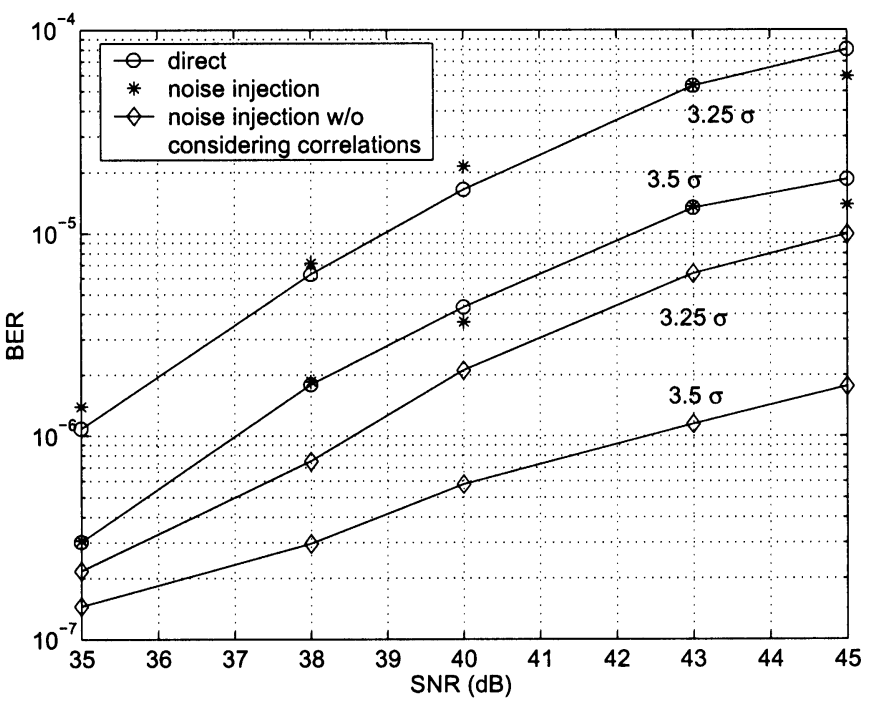

Fig. 3. BER performance of 33-tap optimal-filtering method. Results compare direct simulation with clipping noise-injection method. Results of simulations that do not consider correlation among neighboring samples are also presented to show the significance of correlations.

obtained from the noise-injection method without considering the correlation between neighboring samples. As expected, the BERs obtained by this method are far from the actual BERs obtained from direct simulations. Table I compares the simulation time of direct simulation and the clipping noise injection method. The great difference between the two sets of simulation time, especially for larger values of clipping level, shows the significance of the proposed clipping noise-injection method. We expect larger savings in simulation time for higher values of $A_{\text {clip }}$. Fig. 3 and Table II show similar results for the 33-tap optimal filtering. Again the results show that clipping noise-injection method gives a good approximation of the actual BER of the system and simulation times are reduced significantly. Finally, we note that BER increases with the SNR increase in Figs. 2 and 3 is a consequence of allocation of more bits per block of DMT at higher SNR values, and thus, higher sensitivity to clipping noise.

To assess the performance loss of the system due to reduction in $A_{\text {clip }}$, simulations were carried out using the same channel, but this time, the bit allocation is calculated using channel SNR of $40 \mathrm{~dB}$ and $\mathrm{BER}_{n}$ (BER due to channel noise) of $10^{-5}$. The simulation results for the spectrum-shaping method as well as 33-tap filtering method are shown in Fig. 4. For large values of $A_{\text {clip }}$, where channel noise is dominant, BER is about $10^{-5}$, as one would expect. As $A_{\text {clip }}$ decreases, we observe some perfor-
TABLE II

Simulation Time for Optimal-Filtering Method Before AND AFter USING CLIPPING NOISE INJECTION SNR $(=35 \mathrm{~dB})$

\begin{tabular}{c|c|c}
\hline Clipping Level & Direct Simulation & Noise Injection Method \\
\hline $3.25 \sigma$ & $14 \mathrm{~h} 34 \min 0 \mathrm{~s}$ & $4 \mathrm{~h} 14 \min 20 \mathrm{~s}$ \\
\hline $3.5 \sigma$ & $70 \mathrm{~h} 2 \min 58 \mathrm{~s}$ & $15 \mathrm{~h} 20 \min 9 \mathrm{~s}$ \\
\hline
\end{tabular}

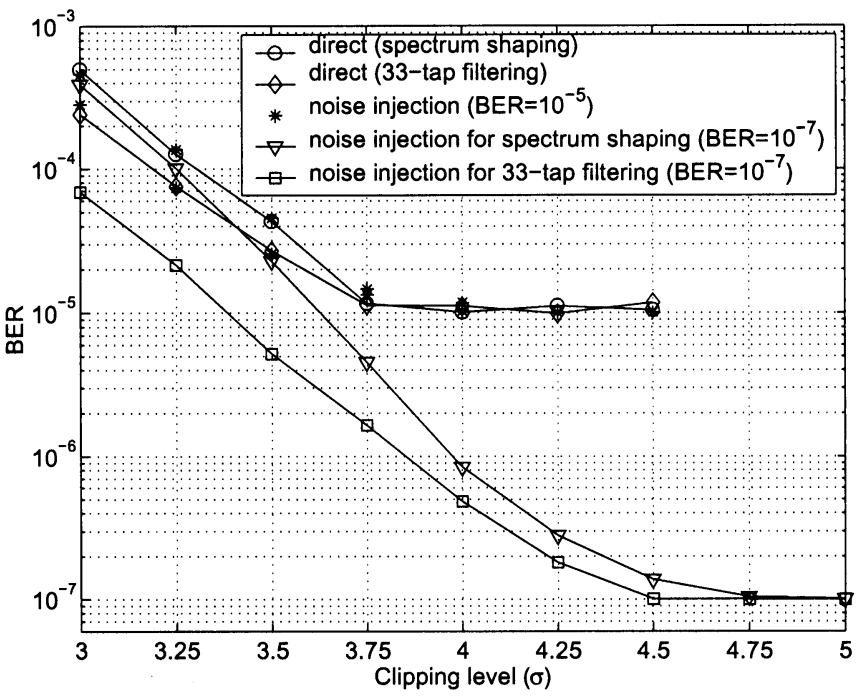

Fig. 4. BER for different clipping levels by direct simulation and noise-injection method.

mance loss in terms of increase in BER. Based on this figure, the system designer can decide on the selection of $A_{\text {clip }}$ for an optimal tradeoff between system complexity and BER performance loss. Note that the setting of BER $=10^{-5}$ in our simulations here is to allow direct simulation, for comparison, in reasonable amount of time. For lower BERs, e.g., $\mathrm{BER}_{n}=10^{-7}$, using direct simulation would require a prohibitively long time to attempt. However, this will not be the case for the clipping noise-injection method. Such results are also shown in Fig. 4.

\section{CONCLUSION}

In this letter, we presented a method called clipping noise injection to provide a fast estimate of the BER performance of DMT-based systems. In particular, we applied the proposed method to estimate the BER of an ADSL system employing spectrum-shaping [2] and optimal-filtering [4] schemes. The results show that while the clipping noise-injection method provides a very good estimation of the BER of the system, it requires significantly less simulation time. This method can be used to estimate the BER of other PAR-reduction schemes for ADSL with proper modifications.

\section{REFERENCES}

[1] M. Jin, B. Farhang-Boroujeny, G. Mathew, and K. C. Indukumar, "A novel fast approach for estimating error propogation in decision feedback detectors," IEEE J. Select. Areas Commun., vol. 19, pp. 668-676, Apr. 2001. 
[2] J. S. Chow, A. C. Bingham, and M. S. Flowers, "Mitigating clipping noise in multicarrier system," in Proc. IEEE Int. Conf. Communications, vol. 2, 1997, pp. 715-719.

[3] W. Yan, "Peak power reduction schemes for ADSL application," M.S. thesis, Nat. Univ. Singapore, Singapore, 2001.

[4] W. Yan, B. Farhang-Boroujeny, and S. Attallah, "Peak power reduction in ADSL using optimal filtering," in Proc. Singapore Int. Conf. Communication Systems 2000, Singapore, Nov. 20-24, 2000, CD-ROM.

[5] — "Clipping noise mitigation methods in DMT-based ADSL systems," in Proc. IEEE Int. Conf. Communications, vol. 6, 2001, pp. $1670-1673$.

[6] J. Tellado and J. M. Cioffi, "Revisiting DMTs Peak-to-Average Ratio," Eur. Telecommun. Standards Inst. (ETSI), TM6, TD08, 1998

[7] A. Gatherer and M. Polley, "Controlling clipping probability in DMT transmission," in Proc. IEEE Asilomar Conf, Signals, Systems, and Computers, vol. 1, 1998, pp. 578-584.
[8] D. J. G. Mestdagh, P. Spruyt, and B. Biran, "Analysis of clipping effect in DMT-based ADSL systems," in Proc. IEEE Int. Conf. Communications, vol. 1, 1994, pp. 293-300.

[9] R. W. Bäuml, R. F. H. Fischer, and J. B. Huber, "Reducing the peak-toaverage power ratio of multicarrier modulation by selected mapping," Electron. Lett., vol. 32, no. 22, pp. 2056-2057, Oct. 1996.

[10] D. J. G. Mestdagh and P. M. P. Spruyt, "A method to reduce the probability of clipping in DMT-based transceivers," IEEE Trans. Commun. vol. 44, pp. 1234-1238, Oct. 1996.

[11] Network and Customer Installation Interfaces-Asymmetric Digital Subscriber Line (ADSL) Metallic Interface, ANSI T1.413, June 1998.

[12] A. Papoulis, Probability, Random Variables, and Stochastic Processes, 3rd ed. New York: McGraw-Hill, 1991.

[13] T. Starr, J. M. Cioffi, and P. Silverman, Understanding Digital Subscriber Line Technology. Upper Saddle River, NJ: Prentice-Hall, 1999. 